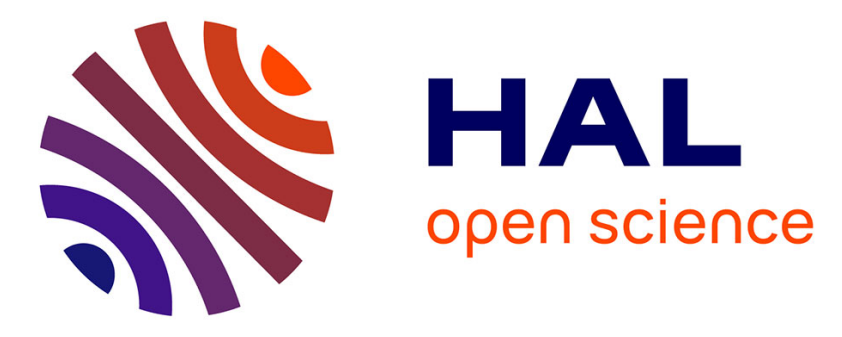

\title{
Testing the link between genome size and growth rate in maize
}

\author{
Maud Tenaillon, Doménica Manicacci, Stephane Nicolas, Francois Tardieu, \\ Claude Welcker
}

\section{> To cite this version:}

Maud Tenaillon, Doménica Manicacci, Stephane Nicolas, Francois Tardieu, Claude Welcker. Testing the link between genome size and growth rate in maize. PeerJ, 2016, 4, pp.e2408. 10.7717/peerj.2408 . hal-01461166

\section{HAL Id: hal-01461166 https://hal.science/hal-01461166}

Submitted on 7 Feb 2017

HAL is a multi-disciplinary open access archive for the deposit and dissemination of scientific research documents, whether they are published or not. The documents may come from teaching and research institutions in France or abroad, or from public or private research centers.
L'archive ouverte pluridisciplinaire HAL, est destinée au dépôt et à la diffusion de documents scientifiques de niveau recherche, publiés ou non, émanant des établissements d'enseignement et de recherche français ou étrangers, des laboratoires publics ou privés. 


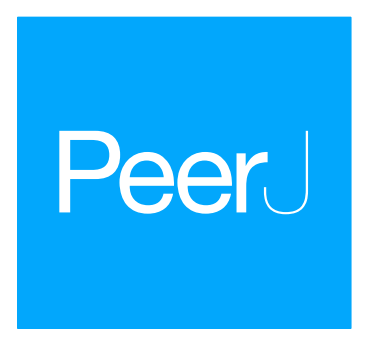

Submitted 26 May 2016

Accepted 4 August 2016

Published 7 September 2016

Corresponding author

Maud I. Tenaillon,

tenaillon@moulon.inra.fr

Academic editor

Jeffrey Ross-Ibarra

Additional Information and Declarations can be found on page 10

DOI 10.7717/peerj.2408

Copyright

2016 Tenaillon et al.

Distributed under

Creative Commons CC-BY 4.0

OPEN ACCESS

\section{Testing the link between genome size and growth rate in maize}

\author{
Maud I. Tenaillon ${ }^{1}$, Domenica Manicacci ${ }^{1}$, Stéphane D. Nicolas ${ }^{1}$, \\ Francois Tardieu ${ }^{2}$ and Claude Welcker ${ }^{2}$ \\ ${ }^{1}$ Génétique Quantitative et Evolution-Le Moulon, INRA-Université Paris-Sud-CNRS—AgroParisTech, \\ Université Paris-Saclay, Gif-sur-Yvette, France \\ ${ }^{2}$ Ecophysiologie des Plantes sous Stress Environnementaux, INRA, Montpellier, France
}

\section{ABSTRACT}

Little is known about the factors driving within species Genome Size (GS) variation. GS may be shaped indirectly by natural selection on development and adaptative traits. Because GS variation is particularly pronounced in maize, we have sampled 83 maize inbred lines from three well described genetic groups adapted to contrasted climate conditions: inbreds of tropical origin, Flint inbreds grown in temperate climates, and Dent inbreds distributed in the Corn Belt. As a proxy for growth rate, we measured the Leaf Elongation Rate maximum during nighttime $\left(\mathrm{LER}_{\max }\right)$ as well as GS in all inbred lines. In addition we combined available and new nucleotide polymorphism data at 29,090 sites to characterize the genetic structure of our panel. We found significant variation for both $\mathrm{LER}_{\max }$ and GS among groups defined by our genetic structuring. Tropicals displayed larger GS than Flints while Dents exhibited intermediate values. $\mathrm{LER}_{\max }$ followed the opposite trend with greater growth rate in Flints than in Tropicals. In other words, $\mathrm{LER}_{\max }$ and GS exhibited a significantly negative correlation $(r=$ $-0.27)$. However, this correlation was driven by among-group variation rather than within-group variation-it was no longer significant after controlling for structure and kinship among inbreds. Our results indicate that selection on GS may have accompanied ancient maize diffusion from its center of origin, with large DNA content excluded from temperate areas. Whether GS has been targeted by more intense selection during modern breeding within groups remains an open question.

Subjects Evolutionary Studies, Plant Science

Keywords Adaptation, Leaf elongation rate, Zea mays, DNA content, Breeding

\section{INTRODUCTION}

It is well established that Genome Size (GS) varies greatly among species, and that much of this variation is caused by repeated sequences (Muñoz Diez et al., 2012; Grover \& Wendel, 2010). There is still, however, a surprising dearth of studies assessing within-species variation. Among plant populations, several investigations have reported GS stability (Ellul et al., 2002; Moscone et al., 2003) while there are a handful of well-documented examples of substantial GS variation (reviewed in Smarda \& Bures, 2010). The extent of within-species GS variation as measured by the coefficient of variation ranges from less than $1 \%$ in Hordeum lechleri (Jakob, Meister \& Blattner, 2004), around 2\% in Arabidopsis thaliana (Long et al., 2013), 3.4\% in Camellia sinensis (Huang et al., 2013) and in Festuca pallens 
(Smarda, Bures \& Horova, 2007), and up to 6\% in maize (Zea mays ssp. mays) and its closest wild relatives (ssp. parviglumis and mexicana), the teosintes (Muñoz Diez et al., 2013).

The factors driving GS variation remain a largely controversial issue. Several competing models have been proposed to explain among-species variations in GS. Interestingly, at least two of these models involve population genetic processes that may drive GS variation within species among populations, and ultimately preside over among-species GS variation (Agren \& Wright, 2011; Petrov, 2001). The "mutational hazard" hypothesis (Lynch et al., 2011) posits that selection to maintain a constant per-genome mutation rate indirectly impacts GS. Providing that selection overcomes drift, the per base-pair-per-generation mutation rate correlates negatively with GS (Sung et al., 2012). Under this model, one expects within-species GS variation to be driven by differences in effective population size that condition the efficiency of natural selection against genome expansion. An alternative hypothesis asserts that positive natural selection may indirectly influence GS variation through developmental or adaptive phenotypes (Knight \& Beaulieu, 2008). In plants, the latter hypothesis has been sustained by a handful of empirical studies demonstrating that GS correlates negatively with development traits such as seedling (Mowforth \& Grime, 1989), root meristem growth rate (Gruner et al., 2010), and cell cycle length (Francis, Davies \& Barlow, 2008). Small genomes indeed presumably facilitate faster cell division and therefore a higher growth rate (Knight, Molinari \& Petrov, 2005; Rayburn, Dudley \& Biradar, 1994).

Improving our understanding of intra-species genome dynamics is essential for elucidating the diversification of GS among related species. Maize is an attractive model to test whether GS is fine-tuned by positive natural selection. Not only does it display the largest within-species GS variation in plants and an exceptional genome fluidity (Chia et al., 2012), but is also characterized by a large effective population size-with estimates ranging from 33,000 (Vigouroux et al., 2002) to $\sim 600,000$ (Gossmann et al., 2010) and 993,000 individuals (Beissinger et al., 2016), and a worldwide distribution with contrasted growing conditions. Actually, maize has a long-lasting history of research on GS variation (for a review, see Knight, Molinari \& Petrov, 2005). The most recent and extensive report on this question in maize landrace populations (Muñoz Diez et al., 2013) has drawn several important conclusions: (1) GS varies primarily among landraces and within-landrace variation is limited; (2) geographical coordinates (altitude, longitude, latitude) are accurate predictors of GS; (3) GS correlates negatively with altitude. These results corroborate significant GS difference between temperate and tropical inbred lines in a sample of 17 improved inbred lines as reported by Chia et al. (2012).

Altogether, these findings suggest that environmental-driven selection on life cycle length and growth rate could indirectly affect GS. To further validate this hypothesis, we measured GS and leaf elongation rate in 83 improved maize inbred lines of various origins in the purpose of establishing a link between GS and growth rate.

\section{MATERIALS AND METHODS}

We have sampled 83 maize inbred lines (inbreds) from the INRA Centre de Ressources Biologiques (Saint Martin de Hinx, France) and from the Maize gene bank at CIMMYT 
in Mexico (Table 1). In order to maximize GS and $\mathrm{LER}_{\max }$ variation, we sampled inbred lines from three of the genetic groups previously defined by Camus-Kulandaivelu et al. (2006): tropical inbreds (Tropicals) characterized by a long life-cycle from sowing to flowering, flint inbreds (Flints) grown in temperate climates with a short life-cycle, and Dent inbreds (Dents) distributed in the Corn Belt with an intermediate life-cycle. Our panel encompassed 50 Tropicals, 18 Flints and 15 Dents.

Genotyping of the 83 inbreds with the Illumina MaizeSNP50 array was either available (Bouchet et al., 2013) or generated for a subset of 11 inbred lines (Data S1). We analyzed 29,090 SNPs contributed by the Panzea project (Zhao et al., 2006) that were developed on a discovery panel of 14 maize and 16 teosinte inbreds. Genotypes of 83 lines on 29,090 SNPs are available in Data S1. We utilized FastStructure v1.0 (Raj, Stephens \& Pritchard, 2014) to evaluate the genetic structure of our sample using $K=2$ and $K=3$ as the number of genetic groups. We determined the memberships of each inbred to the groups at $K=2$ and $K=3$ (Table 1). Kinship was computed from Astle \& Balding (2009) using GenABEL (http://www.genabel.org, Aulchenko, Struchalin \& Van Duijn, 2010).

Plants from each inbred line were characterized for $\mathrm{LER}_{\max }$ in the phenotyping facility Phenodyn (http://bioweb.supagro.inra.fr/phenodyn/) in two experiments (Data S2). The first experiment included all 83 inbred lines with 3 replicated measurements per inbred. The second experiment was a biological replicate for 58 out of the 83 inbred lines, with 3 replicated measurements. Plants were grown in a Klaszmann substrate (30\% clay, $70 \%$ peat) according to the protocol reported in Sadok et al. (2007b). Briefly, the LER $_{\max }$ (in $\mathrm{mm}$ per hour) of the 6th leaf was measured every 15 min during nighttime from 12 to $4 \mathrm{am}$, time at which LER is maximum. Measurements took place in the 4-7 days during which the leaf elongation rate of leaf 6 has no temporal trend over successive nights (Sadok et al., 2007a). A single measure is therefore an average of LER during 4 to 7 nights. Meristem and air temperature, light intensity and air relative humidity, were measured every $15 \mathrm{~min}$. Plants were grown in the greenhouse with naturally fluctuating conditions (200 to $1,100 \mu \mathrm{mol} \mathrm{m}^{-2} \mathrm{~s}^{-1}$ at noon time) under well-watered conditions. During the measurement period, meristem temperature was $18.5^{\circ} \mathrm{C} \pm 0.2^{\circ} \mathrm{C}$ and $20.0 \pm 0.8^{\circ} \mathrm{C}$ in Experiment 1 and 2, respectively. Both soil water potential $(-0.11$ and $-0.15 \mathrm{MPa})$ and vapour pressure deficit $(0.93 \mathrm{kPa} \pm 0.14 \mathrm{kPa}$ and $0.98 \mathrm{kPa} \pm 0.14 \mathrm{kPa})$ were in the range most favorable for growth during measurements.

In parallel, we measured the GS of 3-5 individuals per inbred line-from the same seed lots used for the $\mathrm{LER}_{\max }$ measurements (Data S2). Inbreds were grown in a greenhouse in Gif-sur-Yvette (France) and transferred after 3 weeks to the Imagif facility in Gif-sur-Yvette. The total nuclear DNA amount was assessed by flow cytometry according to Marie \& Brown (1993) Pisum sativum L. 'Long Express' ( $2 C=8.37 \mathrm{pg}$ ) was used as an internal standard. Leaves of the internal standard and maize lines were chopped using a razor blade in a plastic Petri dish with $1 \mathrm{ml}$ of Gif nuclei-isolation buffer $\left(45 \mathrm{mM} \mathrm{MgCl}_{2}, 30 \mathrm{mM}\right.$ sodium citrate, 60 $\mathrm{mM}$ MOPS, $1 \%(\mathrm{w} / \mathrm{v})$ polyvinylpyrrolidone $10,000, \mathrm{pH} 7.2)$ containing $0.1 \%(\mathrm{w} / \mathrm{v})$ Triton $\mathrm{X}-100$, supplemented with $5 \mathrm{mM}$ sodium metabisulphite and RNAse $(2.5 \mathrm{U} / \mathrm{ml})$. The suspension was filtered through $50 \mu \mathrm{m}$ nylon mesh. The nuclei were stained with $50 \mu \mathrm{g} / \mathrm{ml}$ propidium iodide and kept 5 min at $4{ }^{\circ} \mathrm{C}$. DNA content of 5,000-10,000 stained nuclei was 
Table 1 List of inbred lines with measures of Genome Size (GS), LERmax (LER) and membership at $K=2($ Group 1,2$)$ and $K=3$

(Group 1, 2, 3).

\begin{tabular}{|c|c|c|c|c|c|c|c|c|c|c|}
\hline \multirow{2}{*}{$\begin{array}{l}\text { Inbred line } \\
\mathrm{CH} 10\end{array}$} & \multicolumn{2}{|c|}{ GS $(\mathbf{p g})$} & \multicolumn{2}{|c|}{$\operatorname{LER}(\mathbf{m m} / \mathbf{h})$} & \multirow{2}{*}{$\begin{array}{l}\text { K2_G1 } \\
1.000\end{array}$} & \multirow{2}{*}{$\begin{array}{l}\text { K2_G2 } \\
0.000\end{array}$} & \multirow{2}{*}{$\begin{array}{l}\text { K3_G1 } \\
1.000\end{array}$} & \multirow{2}{*}{$\begin{array}{l}\text { K3_G2 } \\
0.000\end{array}$} & \multirow{2}{*}{$\begin{array}{l}\text { K3_G3 } \\
0.000\end{array}$} & \multirow{2}{*}{$\begin{array}{l}\text { K3_group } \\
\text { Flint }\end{array}$} \\
\hline & 5.05 & $(0.026)$ & 6.77 & - & & & & & & \\
\hline EP1 & 5.17 & $(0.129)$ & 4.54 & $(0.149)$ & 0.928 & 0.072 & 0.940 & 0.000 & 0.060 & Flint \\
\hline F39 & 5.31 & $(0.070)$ & 6.25 & $(0.610)$ & 0.879 & 0.121 & 0.894 & 0.000 & 0.106 & Flint \\
\hline F471 & 5.26 & $(0.101)$ & 5.66 & $(0.113)$ & 0.867 & 0.133 & 0.905 & 0.000 & 0.095 & Flint \\
\hline FC16 & 5.27 & $(0.027)$ & 6.83 & - & 0.670 & 0.330 & 0.675 & 0.000 & 0.325 & Flint \\
\hline FC209 & 5.05 & $(0.090)$ & 6.21 & $(0.047)$ & 1.000 & 0.000 & 1.000 & 0.000 & 0.000 & Flint \\
\hline FC24 & 5.41 & $(0.093)$ & 5.99 & $(0.045)$ & 1.000 & 0.000 & 1.000 & 0.000 & 0.000 & Flint \\
\hline FV2 & 5.20 & $(0.069)$ & 5.40 & $(0.251)$ & 1.000 & 0.000 & 1.000 & 0.000 & 0.000 & Flint \\
\hline FV65 & 5.21 & $(0.020)$ & 6.65 & - & 0.868 & 0.132 & 0.876 & 0.000 & 0.124 & Flint \\
\hline FV7 & 5.24 & $(0.055)$ & 6.30 & $(0.514)$ & 1.000 & 0.000 & 1.000 & 0.000 & 0.000 & Flint \\
\hline FV71 & 5.10 & $(0.020)$ & 5.14 & $(0.129)$ & 0.923 & 0.077 & 0.976 & 0.000 & 0.024 & Flint \\
\hline FV75 & 5.11 & $(0.041)$ & 5.86 & $(0.575)$ & 1.000 & 0.000 & 1.000 & 0.000 & 0.000 & Flint \\
\hline FV76 & 5.27 & $(0.089)$ & 5.21 & - & 0.821 & 0.179 & 0.840 & 0.000 & 0.160 & Flint \\
\hline ND30 & 5.04 & $(0.047)$ & 6.94 & - & 1.000 & 0.000 & 1.000 & 0.000 & 0.000 & Flint \\
\hline NY302 & 4.96 & $(0.057)$ & 5.23 & $(0.269)$ & 1.000 & 0.000 & 0.796 & 0.204 & 0.000 & Flint \\
\hline PB40R & 5.28 & $(0.089)$ & 5.04 & $(0.046)$ & 0.770 & 0.230 & 0.725 & 0.087 & 0.187 & Flint \\
\hline W85 & 5.19 & $(0.045)$ & 5.24 & $(0.397)$ & 1.000 & 0.000 & 1.000 & 0.000 & 0.000 & Flint \\
\hline YUBR05 & 5.21 & $(0.073)$ & 4.80 & - & 0.724 & 0.276 & 0.542 & 0.458 & 0.000 & Flint \\
\hline $\mathrm{B} 73$ & 5.21 & $(0.055)$ & 5.42 & $(0.369)$ & 0.490 & 0.510 & 0.000 & 1.000 & 0.000 & Dent \\
\hline CI1872U & 5.26 & $(0.054)$ & 4.73 & (1.046) & 0.305 & 0.695 & 0.000 & 0.729 & 0.271 & Dent \\
\hline EA1433 & 5.24 & $(0.052)$ & 4.21 & $(0.519)$ & 0.416 & 0.584 & 0.206 & 0.420 & 0.373 & Dent \\
\hline FC1852 & 5.33 & $(0.054)$ & 6.12 & $(0.249)$ & 0.494 & 0.506 & 0.000 & 1.000 & 0.000 & Dent \\
\hline FV252 & 5.23 & $(0.199)$ & 4.80 & $(0.108)$ & 0.449 & 0.551 & 0.000 & 1.000 & 0.000 & Dent \\
\hline K64R & 5.24 & $(0.115)$ & 5.68 & $(0.249)$ & 0.313 & 0.687 & 0.052 & 0.538 & 0.410 & Dent \\
\hline KY21 & 5.20 & $(0.045)$ & 5.56 & $(0.885)$ & 0.416 & 0.584 & 0.000 & 1.000 & 0.000 & Dent \\
\hline LAN496 & 5.17 & $(0.050)$ & 5.97 & $(0.009)$ & 0.476 & 0.524 & 0.076 & 0.924 & 0.000 & Dent \\
\hline MBS847 & 5.17 & $(0.008)$ & 4.45 & $(0.519)$ & 0.437 & 0.563 & 0.000 & 1.000 & 0.000 & Dent \\
\hline MO17 & 5.16 & $(0.010)$ & 4.78 & $(0.107)$ & 0.448 & 0.552 & 0.000 & 1.000 & 0.000 & Dent \\
\hline N25 & 5.31 & $(0.056)$ & 4.72 & $(0.377)$ & 0.466 & 0.534 & 0.000 & 1.000 & 0.000 & Dent \\
\hline N6 & 5.22 & $(0.067)$ & 6.28 & - & 0.520 & 0.480 & 0.110 & 0.890 & 0.000 & Dent \\
\hline SC55 & 5.48 & $(0.016)$ & 6.24 & $(0.244)$ & 0.271 & 0.729 & 0.045 & 0.493 & 0.462 & Dent \\
\hline SCMALAWI & 5.45 & $(0.108)$ & 6.44 & $(0.527)$ & 0.263 & 0.737 & 0.000 & 0.609 & 0.391 & Dent \\
\hline W117U & 5.32 & $(0.027)$ & 5.03 & - & 0.423 & 0.577 & 0.000 & 1.000 & 0.000 & Dent \\
\hline A6 & 5.87 & $(0.127)$ & 4.46 & $(0.490)$ & 0.000 & 1.000 & 0.000 & 0.000 & 1.000 & Tropical \\
\hline L256 & 5.32 & $(0.031)$ & 5.63 & $(0.323)$ & 0.460 & 0.540 & 0.465 & 0.000 & 0.535 & Tropical \\
\hline BA90 & 5.41 & $(0.080)$ & 5.54 & $(0.139)$ & 0.366 & 0.634 & 0.201 & 0.356 & 0.443 & Tropical \\
\hline CLA17 & 5.80 & $(0.151)$ & 5.67 & $(0.137)$ & 0.000 & 1.000 & 0.000 & 0.059 & 0.941 & Tropical \\
\hline CML69 & 5.64 & $(0.039)$ & 5.06 & $(0.416)$ & 0.000 & 1.000 & 0.000 & 0.000 & 1.000 & Tropical \\
\hline CML245 & 5.70 & $(0.133)$ & 5.59 & (1.009) & 0.330 & 0.670 & 0.201 & 0.273 & 0.526 & Tropical \\
\hline CML247 & 5.64 & $(0.129)$ & 5.12 & $(0.804)$ & 0.000 & 1.000 & 0.000 & 0.000 & 1.000 & Tropical \\
\hline CML254 & 5.50 & $(0.082)$ & 5.71 & $(0.814)$ & 0.000 & 1.000 & 0.000 & 0.000 & 1.000 & Tropical \\
\hline
\end{tabular}


Table 1 (continued)

\begin{tabular}{|c|c|c|c|c|c|c|c|c|c|c|}
\hline \multirow{2}{*}{$\begin{array}{l}\text { Inbred line } \\
\text { CML287 }\end{array}$} & \multicolumn{2}{|c|}{ GS (pg) } & \multicolumn{2}{|c|}{$\operatorname{LER}(\mathbf{m m} / \mathbf{h})$} & \multirow{2}{*}{$\begin{array}{l}\text { K2_G1 } \\
0.000\end{array}$} & \multirow{2}{*}{$\begin{array}{l}\text { K2_G2 } \\
1.000\end{array}$} & \multirow{2}{*}{$\begin{array}{l}\text { K3_G1 } \\
0.000\end{array}$} & \multirow{2}{*}{$\begin{array}{l}\text { K3_G2 } \\
0.000\end{array}$} & \multirow{2}{*}{$\begin{array}{l}\text { K3_G3 } \\
1.000\end{array}$} & \multirow{2}{*}{$\begin{array}{l}\text { K3_group } \\
\text { Tropical }\end{array}$} \\
\hline & 5.48 & $(0.042)$ & 5.89 & $(0.660)$ & & & & & & \\
\hline CML312 & 5.31 & $(0.073)$ & 4.06 & - & 0.000 & 1.000 & 0.000 & 0.000 & 1.000 & Tropical \\
\hline CML333 & 5.54 & $(0.073)$ & 4.88 & $(0.640)$ & 0.051 & 0.949 & 0.023 & 0.061 & 0.917 & Tropical \\
\hline CML340 & 5.51 & $(0.068)$ & 5.27 & - & 0.000 & 1.000 & 0.000 & 0.000 & 1.000 & Tropical \\
\hline CML341 & 5.50 & $(0.046)$ & 4.53 & - & 0.000 & 1.000 & 0.000 & 0.000 & 1.000 & Tropical \\
\hline CML344 & 5.58 & $(0.092)$ & 3.80 & - & 0.000 & 1.000 & 0.000 & 0.000 & 1.000 & Tropical \\
\hline CML440 & 5.60 & $(0.028)$ & 4.21 & - & 0.063 & 0.937 & 0.063 & 0.000 & 0.937 & Tropical \\
\hline CML91 & 5.44 & $(0.053)$ & 4.60 & $(0.802)$ & 0.109 & 0.891 & 0.032 & 0.149 & 0.819 & Tropical \\
\hline CMLP1 & 5.60 & $(0.087)$ & 4.83 & $(0.020)$ & 0.000 & 1.000 & 0.000 & 0.000 & 1.000 & Tropical \\
\hline CMLP2 & 5.59 & $(0.080)$ & 5.21 & $(0.457)$ & 0.000 & 1.000 & 0.000 & 0.000 & 1.000 & Tropical \\
\hline CZL04006 & 5.51 & $(0.142)$ & 6.33 & - & 0.090 & 0.910 & 0.000 & 0.260 & 0.740 & Tropical \\
\hline CZL0617 & 5.55 & $(0.097)$ & 5.27 & - & 0.000 & 1.000 & 0.000 & 0.000 & 1.000 & Tropical \\
\hline CZL071 & 5.30 & $(0.054)$ & 6.52 & - & 0.089 & 0.911 & 0.028 & 0.119 & 0.853 & Tropical \\
\hline EA1197 & 5.55 & $(0.124)$ & 5.90 & $(0.268)$ & 0.234 & 0.766 & 0.246 & 0.000 & 0.754 & Tropical \\
\hline EA1201 & 5.56 & $(0.164)$ & 5.74 & $(0.492)$ & 0.152 & 0.848 & 0.152 & 0.000 & 0.848 & Tropical \\
\hline EA1866 & 5.44 & $(0.078)$ & 6.47 & $(0.536)$ & 0.234 & 0.766 & 0.237 & 0.000 & 0.763 & Tropical \\
\hline EA1712 & 5.34 & $(0.012)$ & 6.25 & $(0.486)$ & 0.199 & 0.801 & 0.208 & 0.000 & 0.792 & Tropical \\
\hline $\mathrm{F} 2834 \mathrm{~T}$ & 5.44 & $(0.060)$ & 5.14 & $(0.431)$ & 0.245 & 0.755 & 0.136 & 0.224 & 0.640 & Tropical \\
\hline G37 & 5.65 & $(0.096)$ & 4.70 & $(0.010)$ & 0.000 & 1.000 & 0.000 & 0.000 & 1.000 & Tropical \\
\hline DTPWC9-F115 & 5.55 & $(0.072)$ & 5.35 & - & 0.000 & 1.000 & 0.000 & 0.000 & 1.000 & Tropical \\
\hline DTPWC9-F104 & 5.52 & $(0.030)$ & 4.59 & - & 0.000 & 1.000 & 0.000 & 0.062 & 0.938 & Tropical \\
\hline DTPWC9-F31 & 5.65 & $(0.068)$ & 4.09 & - & 0.000 & 1.000 & 0.000 & 0.000 & 1.000 & Tropical \\
\hline DTPYC9-F74 & 5.46 & $(0.092)$ & 5.37 & - & 0.000 & 1.000 & 0.000 & 0.000 & 1.000 & Tropical \\
\hline DTPYC9-F46 & 5.49 & $(0.105)$ & 5.89 & - & 0.000 & 1.000 & 0.000 & 0.018 & 0.982 & Tropical \\
\hline LPSC7-F64 & 5.45 & $(0.004)$ & 4.84 & - & 0.000 & 1.000 & 0.000 & 0.000 & 1.000 & Tropical \\
\hline LPSC7-F71 & 5.41 & $(0.044)$ & 5.49 & - & 0.000 & 1.000 & 0.000 & 0.000 & 1.000 & Tropical \\
\hline LPSC7-F103 & 5.45 & $(0.019)$ & 4.14 & - & 0.000 & 1.000 & 0.000 & 0.000 & 1.000 & Tropical \\
\hline LPSC7-F86 & 5.49 & $(0.084)$ & 4.45 & - & 0.000 & 1.000 & 0.000 & 0.000 & 1.000 & Tropical \\
\hline $\mathrm{H} 16$ & 5.37 & $(0.029)$ & 4.36 & $(0.150)$ & 0.000 & 1.000 & 0.000 & 0.000 & 1.000 & Tropical \\
\hline KUI44 & 5.26 & $(0.101)$ & 4.63 & $(0.823)$ & 0.050 & 0.950 & 0.041 & 0.016 & 0.942 & Tropical \\
\hline KUI11 & 5.54 & $(0.050)$ & 5.58 & $(0.073)$ & 0.000 & 1.000 & 0.000 & 0.042 & 0.958 & Tropical \\
\hline KUI3 & 5.64 & $(0.052)$ & 4.17 & $(0.265)$ & 0.000 & 1.000 & 0.000 & 0.000 & 1.000 & Tropical \\
\hline LP1037 & 5.30 & $(0.037)$ & 6.21 & $(0.678)$ & 0.340 & 0.660 & 0.249 & 0.175 & 0.576 & Tropical \\
\hline LP1233 & 5.39 & $(0.054)$ & 5.97 & $(0.458)$ & 0.240 & 0.760 & 0.243 & 0.000 & 0.757 & Tropical \\
\hline LP35 & 5.40 & $(0.158)$ & 5.69 & $(0.168)$ & 0.243 & 0.757 & 0.242 & 0.008 & 0.750 & Tropical \\
\hline MO22 & 5.45 & $(0.097)$ & 5.40 & $(0.117)$ & 0.069 & 0.931 & 0.065 & 0.000 & 0.935 & Tropical \\
\hline NC298 & 5.75 & $(0.107)$ & 4.77 & $(0.815)$ & 0.000 & 1.000 & 0.000 & 0.000 & 1.000 & Tropical \\
\hline NC304 & 5.48 & $(0.026)$ & 5.02 & $(0.124)$ & 0.000 & 1.000 & 0.000 & 0.000 & 1.000 & Tropical \\
\hline NC320 & 5.40 & $(0.099)$ & 5.61 & $(0.750)$ & 0.210 & 0.790 & 0.000 & 0.465 & 0.535 & Tropical \\
\hline NC338 & 5.78 & $(0.107)$ & 4.98 & $(0.145)$ & 0.000 & 1.000 & 0.000 & 0.000 & 1.000 & Tropical \\
\hline TZI18 & 5.89 & $(0.044)$ & 5.39 & $(0.112)$ & 0.000 & 1.000 & 0.000 & 0.000 & 1.000 & Tropical \\
\hline ZN6 & 5.42 & $(0.036)$ & 5.61 & $(0.527)$ & 0.249 & 0.751 & 0.252 & 0.000 & 0.748 & Tropical \\
\hline
\end{tabular}


determined for each sample using a flow cytometer (CyFlow SL3, Partec-Sysmex. Excitation $532 \mathrm{~nm}, 30 \mathrm{~mW}$; emission through a $630 / 30 \mathrm{~nm}$ band-pass filter). The total 2C DNA value was calculated using the linear relationship between the fluorescent signals from stained nuclei of the maize and the internal standard. We performed three technical replicates per plant. In addition, we employed the inbred line B73 (maize reference genome) to verify the flow cytometer calibration at regular time intervals.

The $\mathrm{LER}_{\max }$ and GS values were averaged among technical replicates (Data S2). LER max of 58 inbred lines replicated over the two experiments were compared using the Bland and Altman's method (1986). The replicates were highly concordant with differences between replicates that did not differ from $0(t=-1.3, d f=28, P=0.20)$, and no correlation between differences between replicates and inbred line mean values $(t=-1.6 ; d f=27$, $P=0.13$ ). GS measurement was replicated on 3-5 plants per line, except for three that were replicated twice and B73 for which we had 14 replicates. Given the high and variable replicates number, the Bland and Altman's method could not be applied. Instead, we performed a one-way ANOVA and showed that GS variation was mainly owed to inbred line differences $\left(R^{2}=89.7 \%\right)$, with only $10.3 \%$ variation across biological replicates. Means and standard deviations for $\mathrm{LER}_{\max }$ and GS across biological replicates for each inbred line are reported in Table 1, and mean values were used for further statistical analyses. All statistical analyses were performed using the R software.

The effect of genetic groups on $\mathrm{LER}_{\max }$ and GS was first tested using linear regression on quantitative memberships obtained from FastStructure. We also employed a one-way ANOVA with a qualitative classification of inbreds as Flints, Dents or Tropicals. In this case, inbreds were assigned to a group based on its highest membership coefficient as determined by FastStructure at $K=3$. We computed pairwise differences between groups using Tukey-Kramer contrasts. We tested the correlation between LER $_{\max }$ and GS first by simple regression; second we corrected for genetic structure by adding qualitative or quantitative memberships obtained from FastStructure as covariates in the linear model; third, we used a mixed model declaring FastStructure quantitative membership as a fixed effect and kinship as a random effect (Yu et al., 2006).

\section{RESULTS}

We assembled a panel of 83 maize inbred lines to test the link between genome size (GS) and the leaf elongation rate $\left(\mathrm{LER}_{\max }\right)$. We extracted genotyping data from 29,090 SNPs and assess genetic structuring of the panel. Our results revealed a clear separation between Tropicals and Flints, while Dents were found as admixed individuals when $K=2$. With $K=3$, the Dent inbreds form a distinct genetic group (Fig. 1).

GS varied between $4.96 \mathrm{pg}$ and $5.89 \mathrm{pg}$ (Table 1) with a coefficient of variation of $3.6 \%$. $\mathrm{LER}_{\max }$ ranged from 3.80 to $6.94 \mathrm{~mm} \mathrm{~h}^{-1}$ (Table 1) with a coefficient of variation of $13.7 \%$. Figure 2 illustrates GS and $\mathrm{LER}_{\max }$ variation within and among the three genetic groups, each inbred being assigned to the genetic group of greatest membership. For both traits, mean values significantly differed among groups (one-way ANOVA, GS : $F_{(2 ; 80)}=$ 52.7, $P=2.510^{-15}$; LER : $\left.F_{(2 ; 80)}=4.47, P=0.014\right)$. Confirming previous observations, Tropicals displayed a larger genome size than Flints (Chia et al., 2012) while Dents exhibited 
A. $K=2$

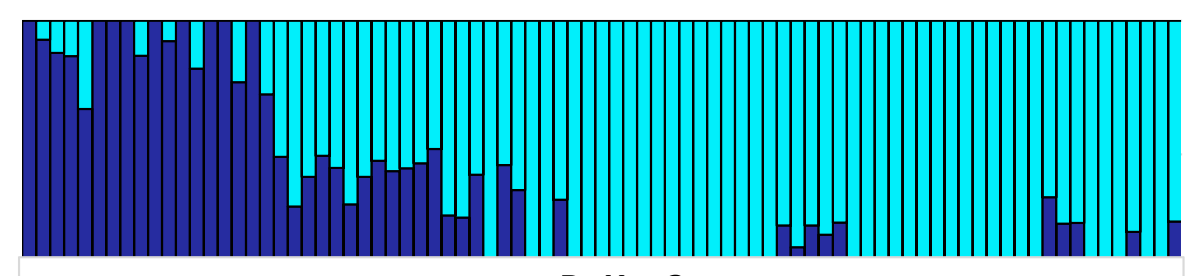

B. $K=3$

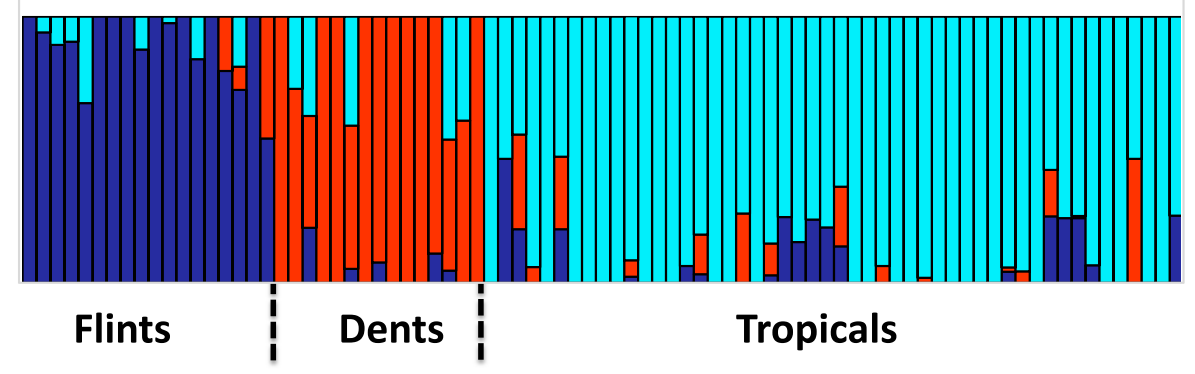

Figure 1 Group membership of 83 maize inbred lines inferred using FastStructure v1.0 (Raj, Stephens \& Pritchard, 2014) from 29,090 SNPs with ancestral group number $K=2$ (A) or $K=3$ (B). The 83 inbred lines are ordered as in Table 1 . Group names were a posteriori defined from the inbred lines with greatest membership with Flints (blue), Dents (red), and Tropicals (cyan).

intermediate GS although non-significantly different from the Flints (Fig. 2A). LER max $_{\text {ax }}$ followed the opposite trend with Flints exhibiting higher values than Tropicals (Fig. 2B). Consistently we found a significant effect of the degree of "Flintness"--membership to the Flint group for $K=2$-on GS (Fig. 2C) and $\mathrm{LER}_{\max }$ (Fig. 2D). The Pearson correlation coefficients were highly significant $\left(r=-0.77, P=2.110^{-17}\right.$ and $r=0.40, P=2.010^{-4}$ respectively for GS and $\left.\mathrm{LER}_{\max }\right)$.

To validate further this pattern, we investigated the correlation between $\mathrm{LER}_{\max }$ and GS and found a significantly negative correlation $\left(r=-0.29, F_{(1 ; 81)}=7.28, P=0.008\right.$, Fig. 3). However, GS may correlate with relatedness among inbreds because measures of closely related inbreds, i.e., those that form a genetic group, are not independent observations. In order to control this effect, we re-analysed the correlation between GS and LER max $_{\text {a }}$ controlling for qualitatively (group assignation from the highest membership coefficient) or quantitatively (group membership coefficient) defined groups. We found that the group effect was significant $\left(F_{(2 ; 77)}=4.68, P=0.012\right)$. Additionally, the correlation was no longer significant when controlling for either qualitative group origin $\left(F_{(1 ; 77)}=1.07, P=0.31\right.$, Fig. 3) or quantitative group membership $\left(F_{(1 ; 77)}=0,003, P=0.95\right)$. As expected when kinship was added to the model, the effect of GS on LER $_{\max }$ remained not significant $(P=0.95)$. The regression slope between GS and LER did not differ among groups as indicated by the non-significant Group X GS interaction on the LER measurements $\left(F_{(2 ; 77)}=2.84, P=0.065\right)$.

Finally, we performed within-group analyses. Sample size was too limited (15 inbreds) to evaluate correlation within Dents. We found no correlation within Flints (18 inbreds). Tropicals (50 inbreds) however exhibited a negative trend, with small genome inbreds 
A.

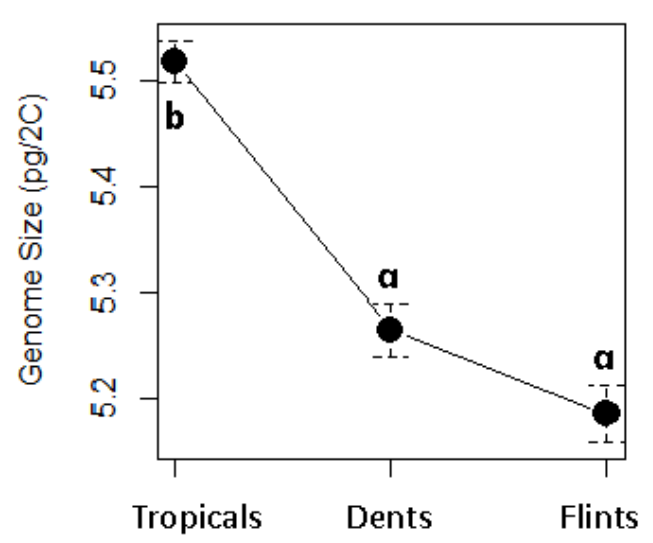

C.

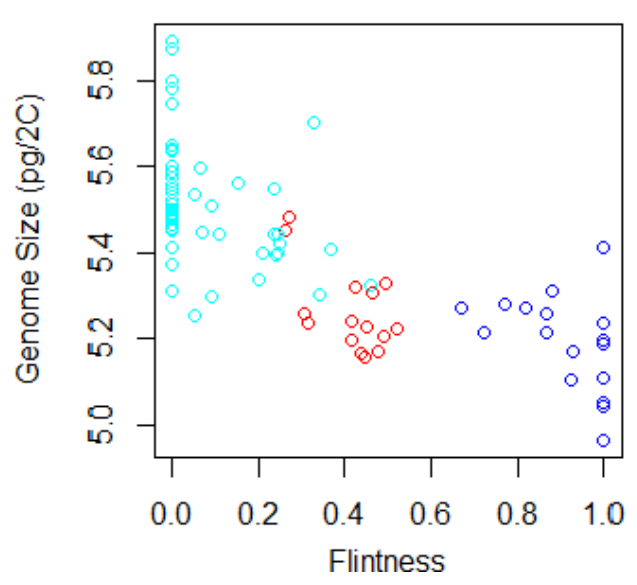

B.
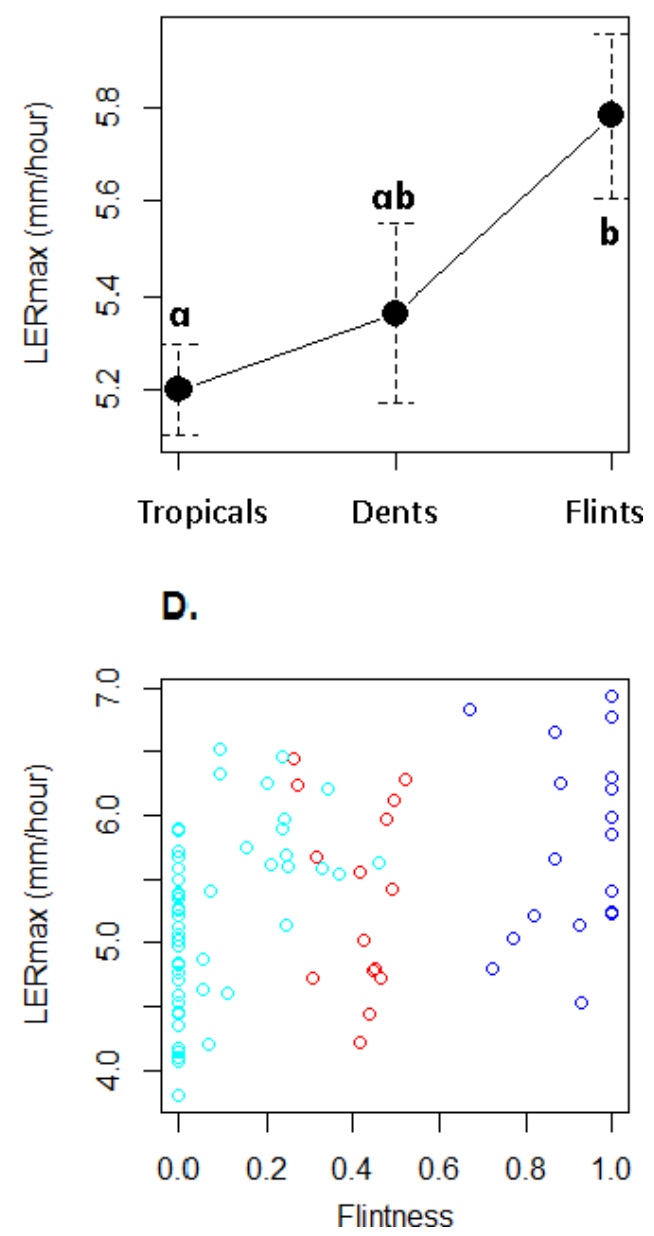

Figure 2 Mean and standard errors across inbred lines for genome size (A) and LERmax (B) for Tropicals, Dents and Flints as defined in Table 1 at $K=3$. Relationship between genome size (C) and LERmax (D) with Flintness as measured by the membership to the Flint group at $K=\mathbf{2}$ (Table 1 ). In (A) and (B), pairs of groups with similar letters exhibit non-significant difference in mean values. In (C) and (D), groups are colored as in Fig. 1.

displaying a tendency towards faster growth rate than larger genome inbreds $(r=-0.26$, $\left.F_{(1 ; 48)}=3.35, P=0.073\right)$.

\section{DISCUSSION}

That plants with smaller genomes may undergo more rapid replication time of their genome, which translates into faster growth rate than plants with larger genomes, is a prediction of the positive natural selection evolution model of genome size. This prediction is based on findings of positive correlation between GS and duration of the cell cycle in 110 angiosperm species (Francis, Davies \& Barlow, 2008). Maize originates from teosintes (Matsuoka et al., 2002) and are characterized by an important range of variation in DNA content (Muñoz Diez et al., 2013). Its genome is extremely fluid (Chia et al., 2012) and 


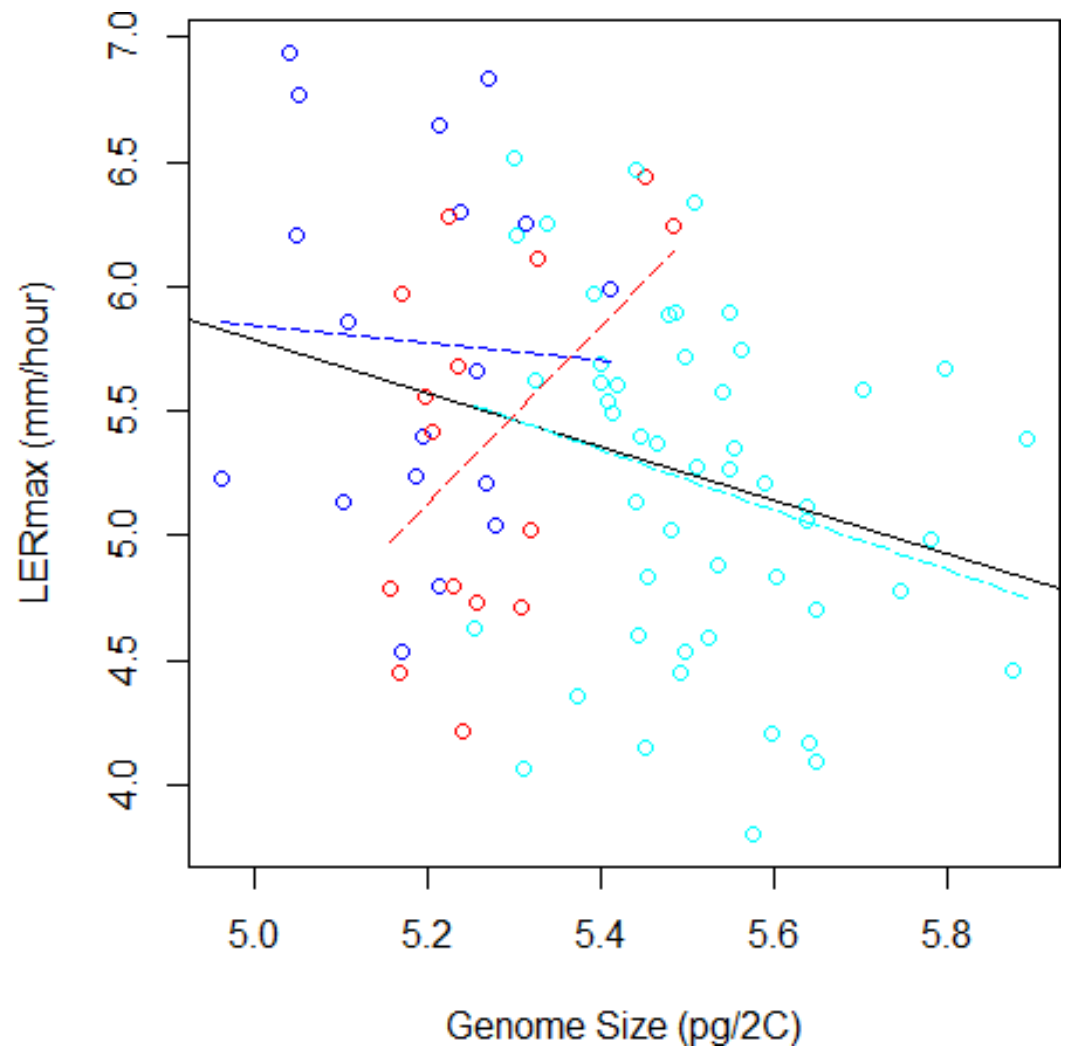

Figure 3 Relation between Genome Size and LERmax within and among groups (Flints in blue, Dents in red, and Tropicals in cyan). The plain line illustrates the linear regression for all data, while colored dotted lines correspond to linear regressions within each group. When the group variable is included in the ANOVA to correct for genetic structure, the relation between LERmax and GS becomes nonsignificant. The Tropical group, that contains 50 inbred lines, displays a tendency for negative correlation between LERmax and GS.

GS may evolve rapidly under selection (Rayburn, Dudley \& Biradar, 1994). Realini et al. (2015) have recently reported a positive correlation between heterochromatin content and length of the vegetative cycle in 9 maize populations sampled from Northeastern Argentina. However a more direct effect of GS variation on growth rate has never been formally tested.

Here, we determined GS and leaf elongation rate $\left(\mathrm{LER}_{\max }\right)$ in 83 improved maize inbred lines selected under contrasted climates. We measured LER $\mathrm{max}_{\max }$ in the developing 6th leaf during the linear phase of elongation, considered as a steady-state (Salah \& Tardieu, 1997). This state is commonly used for measuring cell division and/or tissue expansion (Tardieu et al., 2000). It therefore is a good proxy for growth rate in relation with the timing of cell cycle. Besides, the LER $\mathrm{max}_{\max }$ in maize is reproducible and independent of environmental conditions if corrected for temperature effect (Sadok et al., 2007b). It is also a highly heritable trait (Dignat et al., 2013).

Our sample contained inbred lines from three well-defined genetic groups, the Flints, the Dents and the Tropicals. Genetic structuring analysis based on SNP data (Fig. 1) confirmed previous knowledge on inbreds membership to these groups and the recent history of 
admixture between Tropicals and Flints to form the Dent inbreds at the end of the 19th century (Labate et al., 2003).

Our sample corroborates previous observations from a restricted set of inbreds with temperate inbreds (Flints) exhibiting a significantly smaller GS than tropical (Tropicals) inbreds (Chia et al., 2012) (Figs. 2A and 2C). Interestingly, LER $\max$ followed the opposite trend with Flints exhibiting higher values than Tropicals whether inbred group membership was considered as qualitative (Fig. 2B) or a quantitative trait (Fig. 2D). Note that Dents exhibit intermediate values bot for GS and LER $\max _{\max }$ consistent with their admixed status.

At a first glimpse our results therefore support the hypothesis that smaller genomes exhibit a faster development rate. Because LER $_{\max }$ is a good indicator of growth ability of other organs including reproductive organs (Dignat et al., 2013), it is tempting to speculate that selection for a faster-life cycle in early flowering Flint inbreds has indirectly impacted genome size.

However the negative correlation between GS and LERmax was mainly driven by among-group variation (Fig. 3), suggesting that the existing link between these variables at the origin of the groups was followed by uncorrelated changes during subsequent evolutionary history. Such a pattern has been reported among species, whereby accounting for the phylogenetic history of species altered the relationship between effective population size and GS (Whitney \& Garland, 2010). Noteworthy, within Tropicals smaller genomes displayed a tendency towards faster growth rate than larger genomes. The coefficient of variation of GS was also greater in this group (26\%) than in either Flints (22\%) or Dents (19\%). Tropicals are subjected to high variation in altitude that may exert selective pressure on GS. Additional sampling with limited structuring will be necessary to validate further this result.

Altogether, our results show that selection on GS may have accompanied ancient maize geographical diffusion from its center of origin, consistently with the idea that landraces/inbreds with large DNA content may be excluded from more extreme temperate climates.

\section{ACKNOWLEDGEMENTS}

We are grateful to Jessica Mélique and Spencer Brown for their help in performing the GS measurements and to Spencer Brown for sharing his expertise. We thank Mickaël Bourge and Béatrice Jeunemaitre at the Imagerie-Gif flow cytometry facility for their help in interpreting GS histograms. We also thank Stephane Berthezene and Benoit Suard for their technical support at the phenotyping facility. Delphine Madur handled the samples genotyping. Jean-Tristan Brandenburg provided help with SNP data extraction. Jeff Ross-Ibarra provided helpful comments on the manuscript.

\section{ADDITIONAL INFORMATION AND DECLARATIONS}

\section{Funding}

This work was supported by the Agence Nationale de la Recherche, Investissements d'avenir ANR-10-BTBR-03. The funders had no role in study design, data collection and analysis, decision to publish, or preparation of the manuscript. 


\section{Grant Disclosures}

The following grant information was disclosed by the authors:

Agence Nationale de la Recherche, Investissements d'avenir: ANR-10-BTBR-03.

\section{Competing Interests}

The authors declare there are no competing interests.

\section{Author Contributions}

- Maud I. Tenaillon conceived and designed the experiments, performed the experiments, analyzed the data, contributed reagents/materials/analysis tools, wrote the paper.

- Domenica Manicacci analyzed the data, contributed reagents/materials/analysis tools, prepared figures and/or tables, reviewed drafts of the paper.

- Stéphane D. Nicolas analyzed the data, contributed reagents/materials/analysis tools, reviewed drafts of the paper.

- Francois Tardieu contributed reagents/materials/analysis tools, reviewed drafts of the paper.

- Claude Welcker conceived and designed the experiments, performed the experiments, analyzed the data, contributed reagents/materials/analysis tools, reviewed drafts of the paper.

\section{Data Availability}

The following information was supplied regarding data availability:

The raw data has been supplied as Supplemental Information.

\section{Supplemental Information}

Supplemental information for this article can be found online at http://dx.doi.org/10.7717/ peerj.2408\#supplemental-information.

\section{REFERENCES}

Agren JA, Wright SI. 2011. Co-evolution between transposable elements and their hosts: a major factor in genome size evolution? Chromosome Research 19:777-786 DOI 10.1007/s10577-011-9229-0.

Astle W, Balding DJ. 2009. Population structure and cryptic relatedness in genetic association studies. Statistical Science 24:451-471 DOI 10.1214/09-STS307.

Aulchenko YS, Struchalin MV, Van Duijn CM. 2010. ProbABEL package for genomewide association analysis of imputed data. BMC Bioinformatics 11:134-143 DOI 10.1186/1471-2105-11-134.

Beissinger TM, Wang L, Crosby K, Durvasula A, Hufford MB, Ross-Ibarra J. 2016. Recent demography drives changes in linked selection across the maize genome. Nature Plants 2:16084 DOI 10.1038/nplants.2016.84.

Bland JM, Altman DG. 1986. Statistical method for assessing agreement between two methods of clinical measurements. Lancet 1:307-310. 
Bouchet S, Servin B, Bertin P, Madur D, Combes V, Dumas F, Brunel D, Laborde J, Charcosset A, Nicolas S. 2013. Adaptation of maize to temperate climates: middensity genome-wide association genetics and diversity patterns reveal key genomic regions, with a major contribution of the Vgt2 (ZCN8) locus. PLoS ONE 8:e71377 DOI 10.1371/journal.pone.0071377.

Camus-Kulandaivelu L, Veyrieras JB, Madur D, Combes V, Fourmann M, Barraud S, Dubreuil P, Gouesnard B, Manicacci D, Charcosset A. 2006. Maize adaptation to temperate climate: relationship between population structure and polymorphism in the Dwarf8 gene. Genetics 172:2449-2463 DOI 10.1534/genetics.105.048603.

Chia J-M, Song C, Bradbury PJ, Costich D, De Leon N, Doebley J, Elshire RJ, Gaut B, Geller L, Glaubitz JC, Gore M, Guill KE, Holland J, Hufford MB, Lai J, Li M, Liu X, Lu Y, McCombie R, Nelson R, Poland J, Prasanna BM, Pyhaejaervi T, Rong T, Sekhon RS, Sun Q, Tenaillon MI, Tian F, Wang J, Xu X, Zhang Z, Kaeppler SM, Ross-Ibarra J, McMullen MD, Buckler ES, Zhang G, Xu Y, Ware D. 2012. Maize HapMap2 identifies extant variation from a genome in flux. Nature Genetics 44:803-U238 DOI 10.1038/ng.2313.

Dignat G, Welcker C, Sawkins M, Ribaut JM, Tardieu F. 2013. The growths of leaves, shoots, roots and reproductive organs partly share their genetic control in maize plants. Plant Cell and Environment 36:1105-1119 DOI 10.1111/pce.12045.

Ellul P, Boscaiu M, Vicente O, Moreno V, Rossello JA. 2002. Intra- and interspecific variation in DNA content in Cistus (Cistaceae). Annals of Botany 90:345-351 DOI 10.1093/aob/mcf194.

Francis D, Davies MS, Barlow PW. 2008. A strong nucleotypic effect on the cell cycle regardless of ploidy level. Annals of Botany 101:747-757 DOI 10.1093/aob/mcn038.

Gossmann TI, Song B-H, Windsor AJ, Mitchell-Olds T, Dixon CJ, Kapralov MV, Filatov DA, Eyre-Walker A. 2010. Genome wide analyses reveal little evidence for adaptive evolution in many plant species. Molecular Biology and Evolution 27:1822-1832 DOI 10.1093/molbev/msq079.

Grover E, Wendel JF. 2010. Recent insights into mechanisms of genome size change in plants. Journal of Botany 2010:Article 382732 DOI 10.1155/2010/382732.

Gruner A, Hoverter N, Smith T, Knight CA. 2010. Genome size is a strong predictor of root meristem growth rate. Journal of Botany 2010:390414

DOI 10.1155/2010/390414.

Huang H, Tong Y, Zhang Q-J, Gao L-Z. 2013. Genome size variation among and within Camellia species by using flow cytometric analysis. PLoS ONE 8(5):e64981 DOI 10.1371/journal.pone.0064981.

Jakob SS, Meister A, Blattner FR. 2004. Considerable genome size variation of Hordeum species (Poaceae) is linked to phylogeny, life form, ecology, and speciation rates. Molecular Biology and Evolution 21:860-869 DOI 10.1093/molbev/msh092.

Knight CA, Beaulieu JM. 2008. Genome size scaling through phenotype space. Annals of Botany 101:759-766 DOI 10.1093/aob/mcm321. 
Knight CA, Molinari NA, Petrov DA. 2005. The large genome constraint hypothesis: evolution, ecology and phenotype. Annals of Botany 95:177-190

DOI 10.1093/aob/mci011.

Labate JA, Lamkey KR, Mitchell SE, Kresovich S, Sullivan H, Smith JSC. 2003. Molecular and historical aspects of corn belt dent diversity. Crop Science 43:80-91 DOI 10.2135/cropsci2003.8000.

Long Q, Rabanal FA, Meng D, Huber CD, Farlow A, Platzer A, Zhang Q, Vilhjalmsson BJ, Korte A, Nizhynska V, Voronin V, Korte P, Sedman L, Mandakova T, Lysak MA, Seren U, Hellmann I, Nordborg M. 2013. Massive genomic variation and strong selection in Arabidopsis thaliana lines from Sweden. Nature Genetics 45:884-U218 DOI 10.1038/ng.2678.

Lynch M, Bobay L-M, Catania F, Gout J-F, Rho M. 2011. The repatterning of eukaryotic genomes by random genetic drift. In: Chakravarti A, Green E, eds. Annual review of genomics and human genetics. Vol. 12, 347-366.

Marie D, Brown SC. 1993. A cytometric exercise in plant DNA histograms, with 2Cvalues for 70 species. Biology of the Cell 78:41-51.

Matsuoka Y, Vigouroux Y, Goodman MM, Sanchez GJ, Buckler E, Doebley J. 2002. A single domestication for maize shown by multilocus microsatellite genotyping. Proceedings of the National Academy of Sciences of the United States of America 99:6080-6084 DOI 10.1073/pnas.052125199.

Moscone EA, Baranyi M, Ebert I, Greilhuber J, Ehrendorfer F, Hunziker AT. 2003. Analysis of nuclear DNA content in Capsicum (Solanaceae) by flow cytometry and Feulgen densitometry. Annals of Botany 92:21-29 DOI 10.1093/aob/mcg105.

Mowforth MA, Grime JP. 1989. Intra-population variation in nuclear-DNA amount, cell-size and growth-rate in Poa annua L. Functional Ecology 3:289-295 DOI $10.2307 / 2389368$.

Muñoz Diez C, Gaut BS, Meca E, Scheinvar E, Montes-Hernandez S, Eguiarte L, Tenaillon MI. 2013. Genome size variation in wild and cultivated maize along altitudinal gradients. New Phytologist 199:264-276 DOI 10.1111/nph.12247.

Muñoz Diez C, Vitte C, Ross-Ibarra J, Gaut BS, Tenaillon MI. 2012. Using nextgen sequencing to investigate genome size variation and transposable element content. In: Casacuberta M-AGaJ, ed. Plant transposable elements. Topics in current genetics, vol. 24. Berlin Heidelberg: Springer, 41-58.

Petrov DA. 2001. Evolution of genome size: new approaches to an old problem. Trends in Genetics 17:23-28 DOI 10.1016/S0168-9525(00)02157-0.

Raj A, Stephens M, Pritchard JK. 2014. fastSTRUCTURE: variational inference of population structure in large SNP data sets. Genetics 197:573-U207 DOI 10.1534/genetics.114.164350.

Rayburn AL, Dudley JW, Biradar DP. 1994. Selection for early flowering results in simultaneous selection for reduced nuclear-DNA content in maize. Plant Breeding 112:318-322. 
Realini MF, Poggio L, Camara-Hernandez J, Gonzalez GE. 2015. Intra-specific variation in genome size in maize: cytological and phenotypic correlates. Annals of Botany Plants 8:plv138 DOI 10.1093/aobpla/plv138.

Sadok W, Boussuge B, Welckeir C, Tardieu F. 2007a. A modelling approach to genotype $\mathrm{x}$ environment interaction. Scale and Complexity in Plant Systems Research: GenePlant-Crop Relations 21:77-91 DOI 10.1007/1-4020-5906-X_7.

Sadok W, Naudin P, Boussuge B, Muller B, Welcker C, Tardieu F. 2007b. Leaf growth rate per unit thermal time follows QTL-dependent daily patterns in hundreds of maize lines under naturally fluctuating conditions. Plant Cell and Environment 30:135-146 DOI 10.1111/j.1365-3040.2006.01611.x.

Salah HBH, Tardieu F. 1997. Control of leaf expansion rate of droughted maize plants under fluctuating evaporative demand-a superposition of hydraulic and chemical messages? Plant Physiology 114:893-900 DOI 10.1104/pp.114.3.893.

Smarda P, Bures P. 2010. Understanding intraspecific variation in genome size in plants. Preslia 82:41-61.

Smarda P, Bures P, Horova L. 2007. Random distribution pattern and non-adaptivity of genome size in a highly variable population of Festuca pallens. Annals of Botany 100:141-150 DOI 10.1093/aob/mcm095.

Sung W, Ackerman MS, Miller SF, Doak TG, Lynch M. 2012. Drift-barrier hypothesis and mutation-rate evolution. Proceedings of the National Academy of Sciences of the United States of America 109:18488-18492 DOI 10.1073/pnas.1216223109.

Tardieu F, Reymond M, Hamard P, Granier C, Muller B. 2000. Spatial distributions of expansion rate, cell division rate and cell size in maize leaves: a synthesis of the effects of soil water status, evaporative demand and temperature. Journal of Experimental Botany 51:1505-1514 DOI 10.1093/jexbot/51.350.1505.

Vigouroux Y, Jaqueth JS, Matsuoka Y, Smith OS, Beavis WF, Smith J, Doebley J. 2002. Rate and pattern of mutation at microsatellite loci in maize. Molecular Biology and Evolution 19:1251-1260 DOI 10.1093/oxfordjournals.molbev.a004186.

Whitney KD, Garland Jr T. 2010. Did genetic drift drive increases in genome complexity? PLoS Genetics 6(8):e1001080 DOI 10.1371/journal.pgen.1001080.

Yu J, Pressoir G, Briggs WH, Vroh Bi I, Yamasaki M, Doebley JF, McMullen MD, Gaut BS, Nielsen DM, Holland JB, Kresovich S, Buckler ES. 2006. A unified mixed-model method for association mapping that accounts for multiple levels of relatedness. Nature Genetics 38:203-208 DOI 10.1038/ng1702.

Zhao W, Canaran P, Jurkuta R, Fulton T, Glaubitz J, Buckler E, Doebley J, Gaut B, Goodman M, Holland J, Kresovich S, McMullen M, Stein L, Ware D. 2006. Panzea: a database and resource for molecular and functional diversity in the maize genome. Nucleic Acids Research 34:D752-D757 DOI 10.1093/nar/gkj011. 